\title{
DESIGN STUDY OF STRESSED MIRROR POLISHING (SMP) FIXTURE FOR SEGMENTED MIRROR TELESCOPES
}

\author{
Alikhan Basheer ${ }^{1}$, T. Krishna Murthy ${ }^{2}$ \\ ${ }^{I}$ M-Tech Student, Optical Engineering,Department of Physics, Indian Institute of Space Science and Technology \\ (IIST), Thiruvananthapuram, Kerala, India \\ ${ }^{2}$ Dr. Brahma Prakash Scientist, Applied Optics Area, Laboratory for Electro-Optics Systems (LEOS-ISRO), \\ Bangalore, India
}

\begin{abstract}
Stressed Mirror Polishing (SMP) is a technique developed for the fabrication of off-axis aspheric mirror segments in a rapid and cost effective manner. In SMP, the polishing of the mirror is carried out along with specific loads which are capable of deforming a spherical mirror into any desired aspherical shape and thus reducing the difficulty of aspheric mirror polishing. When the blank is removed from SMP after spherical polish and metrology, the blank will relax to the required aspherical surface with-in desired $P V \& R M S$ surface figure accuracies. This paper discusses the different FEM approaches that can be adopted for simulating the deformations in the mirror blank. For preliminary study purpose, a 36-cm diameter off-axis, meniscus shaped parabolic roundel and a Spherical roundel mirror blanks are taken and required deformations are achieved through various FEM approaches. The net deformed surface is validated and compared with a best fit near spherical surface. Additionally the reversibility of the technique is also proved with-in required Surface accuracies. It is felt necessary that these preliminary simulations should form the basis in understanding the warping process and their locations, so that full SMP fixture can be designed which is capable of warping the mirrors.
\end{abstract}

Keywords: Stressed Mirror Polishing, Segmented Primary Mirror, Aspheric Segment Fabrication, Bending Fixture

\section{INTRODUCTION}

It is proved that SMP technique is one of the optimum approaches for bulk production of thin mirrors, by warping them and polishing them as spherical blanks. In SMP, the polishing of the mirror is carried out along with specific loads which are capable of deforming a spherical mirror into any desired aspherical shape and thus reducing the difficulty of aspheric mirror polishing. Thus the SMP fixture can be used to deform a mirror blank, polish the surface as a spherical surface, and carry out metrology for validation. When the blank is removed from SMP fixture after spherical polish and metrology, the blank should relax to a required aspherical surface with-in desired PV \& RMS surface figure accuracies. Typically the SMP fixture may have to produce warping capability up to $185 \mu \mathrm{m}-215 \mu \mathrm{m}$, yet producing elastic behavior in the glass blank to be polished and in the relaxed state the aspherical surface must be with-in 2 micron (rms) surface. If the deforming range is made large but within limits of elastic behavior, then the same SMP fixture can be tuned to all type of segments with different off axis distances and different aspheric coefficients of a given primary mirror assembly. The forces and bending moments required in the SMP to warp a given glass blank can be calculated through closed form solutions developed for plate bending theories or more precisely through FEM (Finite Element Modeling) approaches. The SMP can be configured with force / displacement actuators located optimally at the rear side or at the edges of the glass blank so that required warping can be achieved.

\section{WARPING THEORY OF SMP}

The method to represent each of the segment surfaces in local coordinate system $(\mathrm{x}, \mathrm{y}, \mathrm{z})$ with respect to the global coordinate system $(\mathrm{X}, \mathrm{Y}, \mathrm{Z})$ is given by Jerry Nelson and Temple-Raston [3].The segment coordinate system can be represented in the global coordinate system by a translation $\Delta \mathrm{Z}$ and a rotation $\varphi$ as shown in Figure 1.

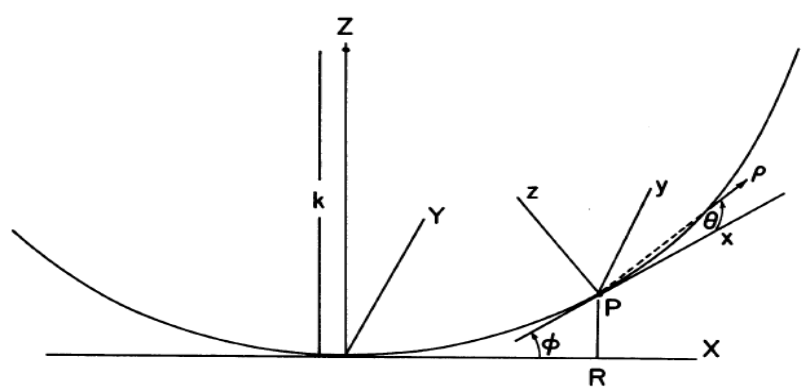

Fig-1: Local coordinate system within the Global coordinate system

In this orientation Z-axis is normal to the Parent mirror surface and $\mathrm{z}$-axis normal to the corresponding segment surface. By considering the segments along radial axis, we can make the orthogonal terms to vanish; so that the general form to represent a surface is reduced in such a way that each term of the segment shape can be represented by a single coefficient termed as polar monomial coefficient (SEG). The segment surface can be represent in the segment coordinate system by [5]: 
$z(\rho, \theta)=\sum_{m, n} \alpha_{m, n}^{S E G} \rho^{m} \cos n \theta \quad m \geq n \geq 0, m-n:$ even

$\alpha_{2,0}{ }^{S E G}=\frac{a^{2}}{k}\left(\frac{2-K \varepsilon^{2}}{4\left(1-K \varepsilon^{2}\right)^{3 / 2}}\right)$

$\alpha_{2,2}^{S E G}=\frac{a^{2}}{k}\left(\frac{K \varepsilon^{2}}{4\left(1-K \varepsilon^{2}\right)^{3 / 2}}\right)$

$\alpha_{3,1}{ }^{S E G}=\frac{a^{3}}{k^{2}}\left(\frac{K \varepsilon\left[1-(K+1) \varepsilon^{2}\right]^{1 / 2}\left(4-K \varepsilon^{2}\right)}{8\left(1-K \varepsilon^{2}\right)^{3}}\right)$

$\alpha_{3,3}{ }^{S E G}=\frac{a^{3}}{k^{2}}\left(\frac{K^{2} \varepsilon^{3}\left[1-(K+1) \varepsilon^{2}\right]^{1 / 2}}{8\left(1-K \varepsilon^{2}\right)^{3}}\right)$

$\alpha_{4,0}{ }^{S E G}=\frac{a^{4}}{k^{3}}\left(\frac{8(1+K)-24 K \varepsilon^{2}+3 K^{2} \varepsilon^{4}(1-3 K)-K^{3} \varepsilon^{6}(2-K)}{64\left(1-K \varepsilon^{2}\right)^{9 / 2}}\right)$

$\alpha_{4,2}{ }^{S E G}=\frac{a^{4}}{k^{3}}\left(\frac{K \varepsilon^{2}\left[2(1+3 K)-(9+7 K) K \varepsilon^{2}+(2+K) K^{2} \varepsilon^{4}\right]}{16\left(1-K \varepsilon^{2}\right)^{9 / 2}}\right)$

$\alpha_{4,4}{ }^{S E G}=\frac{a^{4}}{k^{3}}\left(\frac{K^{2} \varepsilon^{4}\left[1+5 K-K \varepsilon^{2}(6+5 K)\right]}{64\left(1-K \varepsilon^{2}\right)^{9 / 2}}\right)$

In general,

$\alpha_{m, n}{ }^{S E G}=O\left(\frac{a^{m} \varepsilon^{n}}{k^{m-1}}\right)$

where $K=$ the conic constant, $k=$ the parent mirror paraxial ROC, $R=$ the off-axis distance of the segment center, $\varepsilon=R / k$,

the off-axis factor, and $a=$ the segment radius. The stressing coefficients correspond to the amount of stress required to convert a best fit spherical surface to the desired aspheric shape. If $k_{B}$ is the best fit ROC of the blank in its stress-free state, then the stressing coefficients are [5]:

$$
\begin{aligned}
& \alpha_{20}^{\text {STR }}=a^{2} / 2 k_{B}-\alpha_{20}^{\text {SEG }} \text { (Power) } \\
& \alpha_{22}{ }^{S T R}=-\alpha_{22}{ }^{S E G} \\
& \alpha_{31}{ }^{S T R}=-\alpha_{31}{ }^{S E G} \\
& \alpha_{33}{ }^{\text {STR }}=-\alpha_{33}{ }^{S E G} \\
& \alpha_{40}{ }^{S T R}=a^{4} / 8 k_{B}{ }^{3}-\alpha_{40}{ }^{S E G} \quad(\text { Spherical }) \\
& \alpha_{42}{ }^{S T R}=-\alpha_{42}{ }^{S E G} \\
& \alpha_{44}{ }^{S T R}=-\alpha_{44}{ }^{S E G}
\end{aligned}
$$

With the advancement in Stressed Mirror Polishing, it is proved that any desired surface shapes can be achieved by the application of appropriate, continuously varying, tangential moments and shear (normal) forces only around the perimeter of the part. No warping loads are required in the interior of the part. The method for calculating the continuous moment and shear force densities required around a circular segment perimeter to achieve the desired warping is given by Nelson et. al. ${ }^{[1,2]}$. Expressions to find out these Continuous moment density and shear force density from the Polar monomial coefficients are summarized in Table: 1 .

Table 1. Polar Monomial Coefficient relationships

\begin{tabular}{|c|c|c|c|}
\hline Description & Polar Monomial Coefficients & Continuous moment density & Continuous shear force density \\
\hline & $\alpha_{\mathrm{mn}}$ & $\mathrm{M}_{\mathrm{mn}}$ & $\mathrm{V}_{\mathrm{mn}}$ \\
\hline Power & $\alpha_{20}$ & $\left(2 D / a^{2}\right)(1+v) \alpha_{20}^{\text {STR }}$ & $2 M_{22} / a$ \\
\hline Astigmatism & $\alpha_{22}$ & $\left(2 D / a^{2}\right)(1-v) \alpha_{22}^{\text {STR }}$ & $-M_{31} / a$ \\
\hline Coma & $\alpha_{31}$ & $\left(2 D / a^{2}\right)(3+v) \alpha_{31}^{S T R}$ & $-8 M_{33} / a$ \\
\hline Trefoil & $\alpha_{33} / a(3+\mu)$ \\
\hline $\begin{array}{c}\text { Primary } \\
\text { Spherical }\end{array}$ & $\alpha_{40}$ & $\left(6 D / a^{2}\right)(1-v) \alpha_{33}^{\text {STR }}$ & $(-1+\mu) M_{42} / a$ \\
\hline Secondary Astigmatism & $\left.\alpha_{42} / a^{2}\right)(3+v) \alpha_{40}^{S T R}$ & $4 M_{44} / a$ \\
\hline Quadrafoil & $\alpha_{44}$ & $\left(12 D / a^{2}\right) \alpha_{42}^{S T R}$ & $\left(12 D / a^{2}\right)(1-v) \alpha_{44}^{S T R}$ \\
\hline
\end{tabular}

For a given no. of equally angular separated levers (N) with an angle of separation $\theta_{\mathrm{L}}(\mathrm{L}=1$ to $\mathrm{N})$, the discrete moment and shear force required at each lever can be calculated by [5]:

$M_{L}\left(\theta_{L}\right)=\frac{2 \pi a}{N} \sum_{m n} M_{n m} \cos \left(n \theta_{L}\right) \quad$ for even $m-n$, and $m \geq n \geq 0$

$V_{L}\left(\theta_{L}\right)=\frac{2 \pi a}{N} \sum_{m n} V_{n m} \cos \left(n \theta_{L}\right) \quad$ for even $m-n$, and $m \geq n \geq 0$

where $M_{m n}$ and $V_{m n}$ are the continuous moment density and shear force density respectively as given in Table 1 . And $D$ is the plate stiffness modulus given by [1]:

$$
D=E h^{3} / 12\left(1-v^{2}\right)
$$

in which $E$ and $v$ are Young's modulus and Poisson's ratio respectively.

The generation of bending moments and shear forces can be simultaneously achieved in a simple mechanism of a system of weights, levers, and fulcra. If a Radial arm is bonded to the outer cylindrical surface of the mirror blank (typically at 
the middle of the edge thickness / or more appropriately in the neutral axis of the blank) and forces are applied through levers on to the radial arm of the blank as shown in Fig. 2, they will produce both bending moments and shear forces simultaneously by the mechanism. Multiple such lever mechanism may be bonded to the mirror blank around the mirror periphery at equi-spaced. In the present analysis and simulations 24 units of such lever mechanism are found to be optimum to deform the blank to given surface profile either spherical to Parabolic or parabolic to Spherical.

From the above Fig. 2, applying the equilibrium condition, the Shear Force \& Bending Moment is calculated as:

Shear Force, $V_{L}\left(\theta_{L}\right)=F_{1}+F_{2}$

Bending Moment, $M_{L}\left(\theta_{L}\right)=F_{1} r_{1}+F_{2} r_{2}$
Using the above equilibrium condition the $F_{1} \& F_{2}$ are derived for the proposed geometry, $r_{l}=60 \mathrm{~mm} \& r_{2}=300 \mathrm{~mm}$ and are given in Table: 2

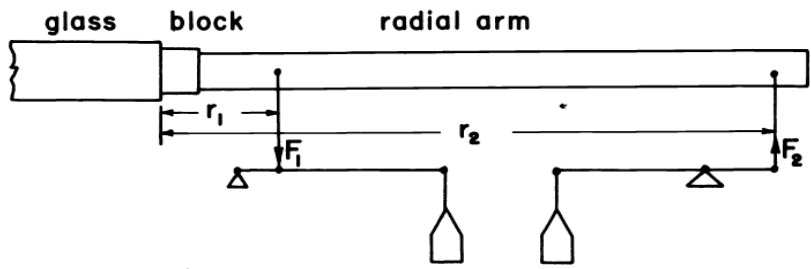

Fig-2: Typical lever mechanism to produce bending moments and shear forces [4]

Table 2. Computed Warping Forces

\begin{tabular}{|c|c|c|c|c|c|c|c|}
\hline $\begin{array}{c}\text { Arm } \\
\text { No. }\end{array}$ & Angle $(\theta \mathrm{L})$ & $\begin{array}{c}\text { Shear } \\
\text { Force, VL, } \\
\mathrm{Kg}\end{array}$ & $\begin{array}{c}\text { Bending } \\
\text { Moment ML, } \\
\text { Kg-cm }\end{array}$ & $\begin{array}{c}\text { Warping } \\
\text { Force(F1), Kg- } \\
\mathrm{cm}\end{array}$ & $\begin{array}{c}\text { Warping } \\
\text { Force(F2), Kg- } \\
\mathrm{cm}\end{array}$ & F1, N & F2, N \\
\hline 1 & 0 & -16.4 & 276.73 & 32.03041 & 15.63041 & 313.8980 & 153.1780 \\
\hline 2 & \pm 15 & -16.78 & 260.38 & 31.82416 & 15.04416 & 311.8768 & 147.4328 \\
\hline 3 & \pm 30 & -17.73 & 213.92 & 31.07583 & 13.34583 & 304.5431 & 130.7891 \\
\hline 4 & \pm 45 & -18.73 & 144.59 & 29.4370 & 10.70708 & 288.4834 & 104.9294 \\
\hline 5 & \pm 60 & -19.07 & 62.68 & 26.44916 & 7.379166 & 259.2018 & 72.31583 \\
\hline 6 & \pm 75 & -18.06 & -20.54 & 21.71916 & 3.659166 & 212.8478 & 35.85983 \\
\hline 7 & \pm 90 & -15.29 & -95.13 & 15.14875 & -0.14125 & 148.4577 & -1.38425 \\
\hline 8 & \pm 105 & -10.73 & -154.32 & 6.9825 & -3.7475 & 68.4285 & -36.7255 \\
\hline 9 & \pm 120 & -4.86 & -195.48 & -2.07 & -6.93 & -20.286 & -67.914 \\
\hline 10 & \pm 135 & 1.46 & -219.94 & -10.98916 & -9.52917 & -107.694 & -93.3858 \\
\hline 11 & \pm 150 & 7.11 & -231.82 & -18.54666 & -11.4367 & -181.757 & -112.079 \\
\hline 12 & \pm 165 & 11.03 & -236.22 & -23.63 & -12.6 & -231.574 & -123.48 \\
\hline 13 & 180 & 12.43 & -237.17 & -25.41958 & -12.9896 & -249.111 & -127.298 \\
\hline
\end{tabular}

\section{MIRROR CONFIGURATION}

As an initial theoretical bench mark study, a smaller size off-axis primary mirror segment of Concave Parabola of $360 \mathrm{~mm}$ diameter with a paraxial Radius of Curvature (ROC) of $3688 \mathrm{~mm}$ and a blank thickness of $25.4 \mathrm{~mm}$ is taken up. The motivation for the selecting such smaller size blank is also due to establish warping force computation methodologies by closed form equations and bench mark the results with the published results in a most valuable and pioneering research paper [2].

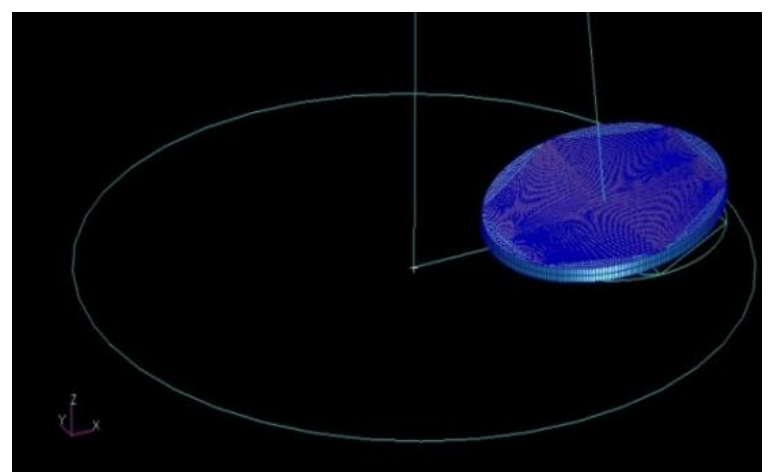

Fig-3: Roundel at the off axis distance

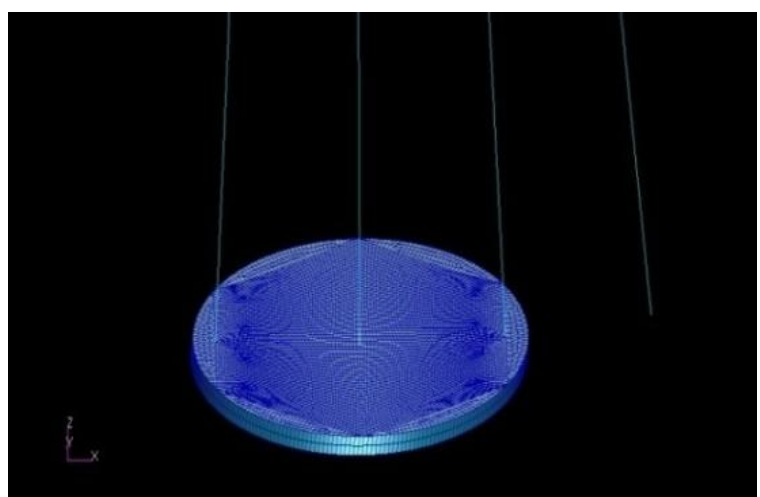

Fig-4: Roundel made into On-axis by rotation about the ROC point

Further by carrying out FEM Analysis with the obtained closed form results the deformed shapes can be simulated and evaluated. In order to achieve desired shape as accurately as possible with that that of theoretically required shape under that action of forces, several FEM simulations are carried out with different load cases. The amount of warping required is based on the difference between the spherical surface to be polished into the deformed surface and the desired aspherical surface over the full aperture as given by Lubliner and Nelson [1] 


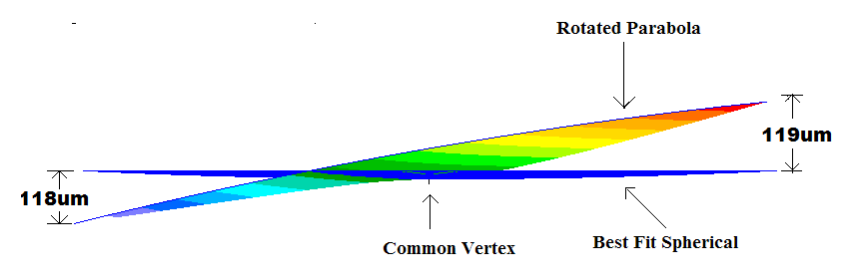

Fig-5: Deviation between parabola and its best fit spherical

The objective of the study is to convert a best fit spherical surface to a near parabolic surface mirror by that application of either Stress or Displacement method using the actuators. The assumption is that, when the roundel is polished to such a near best fit spherical along with the SMP with those specified forces, and when the roundel is removed from SMP, we should get desired parabolic surface with in required accuracy. The amount of warping required is the difference between best fit sphere and the desired parabolic aspheric surface. FEM based approach is consider for establishing and validating the above assumptions.

For the present study, basically two different approaches are adopted.

1. Stress method: In this approach 24 lever system are connected to the mirror and by the application of either bending moment and shear force along with a central support, the mirror is warped to desired shape with different load cases.

2. Displacement method: In this approach, the actuators connected at the bottom of the mirror are displaced by the required amount and this displacement causes deformations on the mirror as actuation.

\section{FEM ANALYSIS}

\subsection{FEM Analysis Using Displacement Method}

Load case 1: Effect of applying Displacement method on actuators

In this particular model, the rear side of the mirror blank is connected with a 24 equally spaced stainless steel actuators which are in turn fixed at the bottom with all six degrees of freedom. Additionally a central support is also given to the mirror blank by an actuator for structural rigidity of the model. From the front surface data of best fit spherical and parabolic surface acquired from the model, the deviation required to transform this best fit spherical to parabolic surface is calculated, and applying the force required to achieve this deviation on the actuators, the desired deviation and finally the desired surface is expected

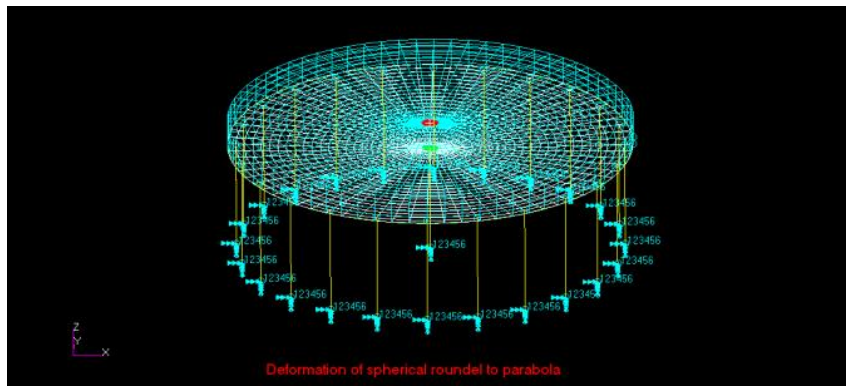

Fig-6: Application of Loads and Boundary Conditions

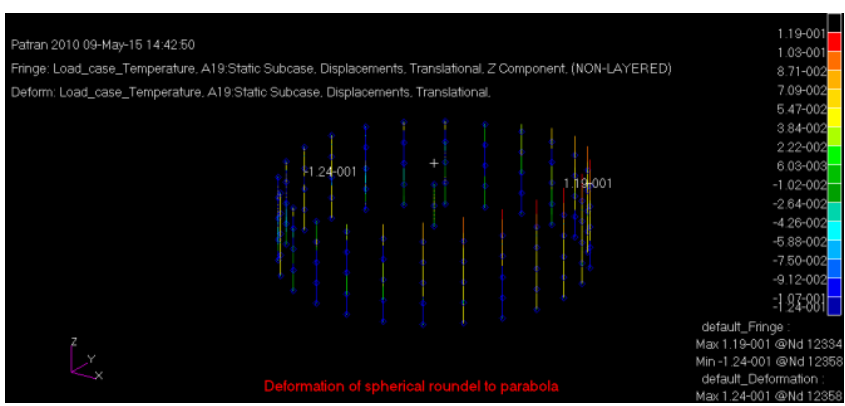

Fig-7: Deformation of actuators due to the application of load

\subsection{FEM Analysis Using Stress Method}

\section{Load case 2: Effect of Bending Moment \& Central support}

The Bending Moments as computed from closed form equations and given in Table: 2 are applied through 24 units of lever assemblies around periphery of the mirror and are shown in Fig.7. As shown in the above FE model, the levers are simulated as beam elements of SS material. As per geometry, each lever length is taken as $300 \mathrm{~mm}$ projecting outward from mirror blank outer periphery. Forces acting perpendicular to radial arm and acting along gravity vector viz. "F1" is applied at $60 \mathrm{~mm}$ from the mirror and similarly "F2" is applied at $300 \mathrm{~mm}$ i.e. outer end of the lever. To simulate the bonding of the lever at the mirror edge, Multi Point Constraint (MPC) mechanism is used where nine nodes on the periphery of the mirror covering an area approximately $150 \mathrm{~mm}$ sq. are linked to the master node of the radial arm.

The vertical Force arms of length $30 \mathrm{~mm}$ are linked to the radial arm as shown in Fig.6. The warping forces "F1" \& "F2" given in Table: 2 together will produce the Bending movements on to the mirror blank according to the plate bending theory. The forces "F1" \& "F2" are that for the deformation of a spherical to anti-parabola on SMP so that after polishing a sphere in the deformed surface and removing the loads; required parabolic surface is obtained. However, in our present simulations, the objective is to deform spherical surface and compares the deformed surface directly with that of required surface (Parabolic) and vice versa. Hence the forces computed in Table: 2 are reversed in sign keeping the magnitude same. This facilitates direct one to one comparison of the deformed surface vs desired surface. 


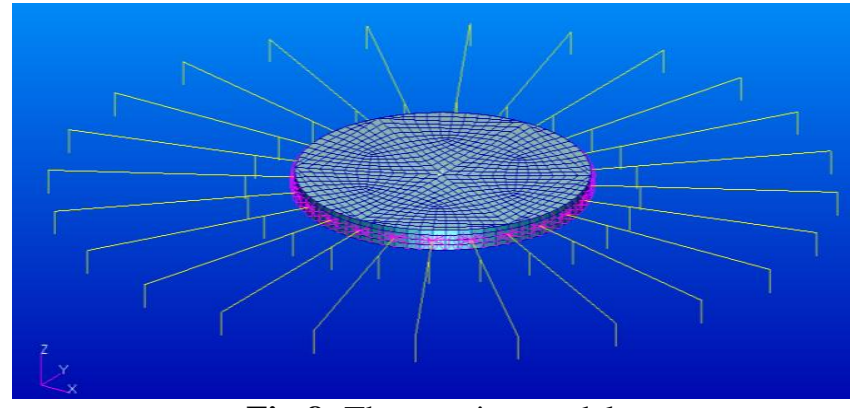

Fig-8: The warping model

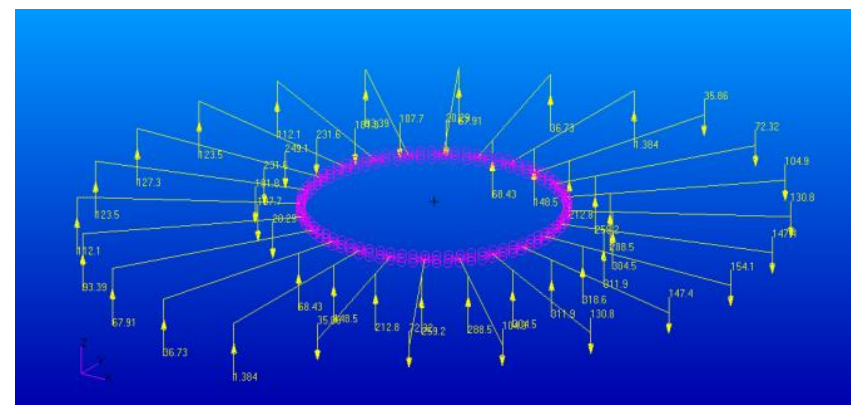

Fig-9: The warping model with Loads applied

\section{Load case 3: Effect of Shear Force \& Central Support}

The shear forces as computed from closed form equations and given in Table: 2 are applied through 24 axial arms linked to the radial arm in two different methods. In one method the application of force is at the interface junction of the radial arm but without radial arms as shown in Fig. 8. and in the other one, the application of force is at the rear edge of the mirror blank as shown in Fig. 9. As explained, the shear forces calculated in the Table: 2. are for deforming the spherical surface to anti-parabola on SMP so that after polishing a sphere in the deformed surface and removing the loads, the required parabolic surface is obtained.

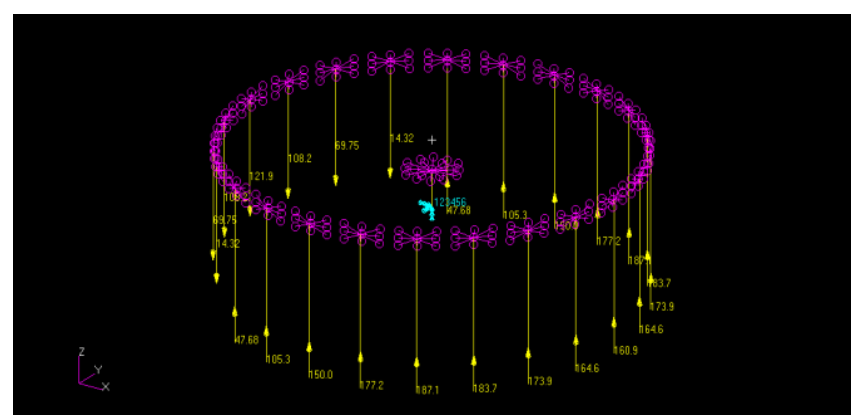

Fig-10: Application of Shear Forces on cylindrical rim

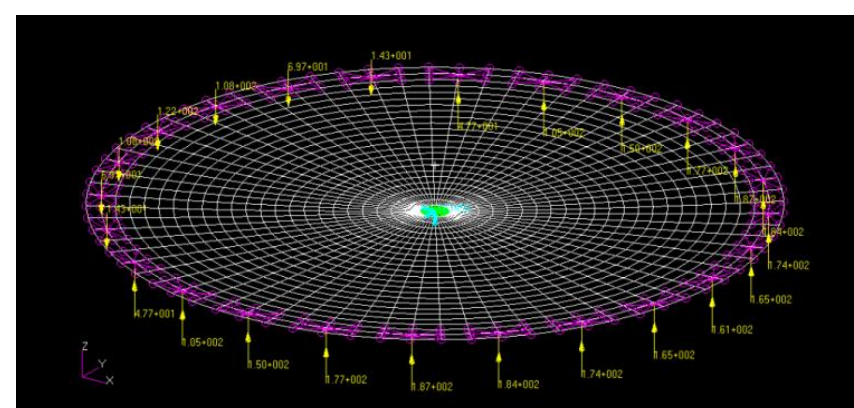

Fig-11: Application of Shear Forces on rear side

\subsection{FEM Analysis To Prove Reversibility of SMP}

After finding out the optimum approach for the SMP in terms of surface RMS, the same model has taken up to prove the reversibility of the technique.

Exercise 1: Study of deformation of a spherical blank into parabolic.

Exercise 2: Study of deformation of a spherical blank into anti-parabolic.

Exercise 3: Study of deformation of a parabolic blank into spherical.

In Ex: 1, a spherical blank is deformed in to a parabolic surface and the surface RMS in terms of Fringe Zernike Coefficients is noted down. After that, the same spherical blank is deformed into an anti-parabola by simply reversing the direction of the applied shear forces and the surface RMS is noted down. This RMS need not to be same as the previous one, because, one is deformed into entirely opposite curvature shape, so that variation should be there in the results. Next in Ex: 3, a parabolic mirror is taken up and deformed into a spherical by reversing the direction of shear force in Exercise: 1, and the surface RMS is noted down. This result is expected to be match very close to the Ex: 1 .

\subsection{FEM analysis to modify the results}

After obtaining the optimum approach, by examining the variation in the deviations achieved, the shear forces can be modified at required locations to further modification of the results. It can be also achieved by reducing certain aberration terms like astigmatism. So an exercise has done in order find out the effect of reducing the astigmatic term by gradual modification of shear force at $90^{\circ}$ and $270^{\circ}$ region alone. For this purpose the Zernike coefficients are found out by increasing and decreasing the Shear force by $10 \%$. It is observed that an $8 \%$ improvement in the surface RMS by the reduction of shear force.

Table 3: Surface RMS for the Modified shear force

\begin{tabular}{|c|c|c|c|}
\hline \multicolumn{5}{|c|}{ Obtained Zernike Coefficients } \\
\hline NAME & $\begin{array}{c}\text { Normal } \\
\text { Shear Force }\end{array}$ & $\begin{array}{c}\text { Incr. Shear } \\
\text { Force }\end{array}$ & $\begin{array}{c}\text { Decr. Shear } \\
\text { Force }\end{array}$ \\
\hline Piston & 21.31635521 & 22.86467943 & 20.05157003 \\
\hline Tilt x & 252.086607 & 255.8882068 & 250.5923036 \\
\hline Tilt y & -0.00113314 & -0.00014837 & -0.0001893 \\
\hline Focus & 16.9461829 & 18.12255164 & 15.89161284 \\
\hline Astig x & -15.5533248 & -17.6828588 & -13.5865425 \\
\hline Astig y & -0.0012617 & 0.00100024 & 0.0009485 \\
\hline Coma x & 13.64603523 & 13.77238198 & 13.48801768 \\
\hline Coma y & -0.00129461 & -0.00012469 & -0.00010839 \\
\hline Pri. sph & -3.08825752 & -3.317479 & -2.90813654 \\
\hline Trefoil x & 0.03863643 & -0.1151083 & 0.23183067 \\
\hline Trefoil y & -0.0029657 & 0.00114941 & 0.00115775 \\
\hline Sec. Astig x & 0.50836662 & 0.59222338 & 0.45830571 \\
\hline Sec. Astig y & -0.00260173 & -0.00000474 & 0.00000833 \\
\hline Sec. Coma x & -6.64346892 & -6.58477973 & -6.44864238 \\
\hline Sec. Coma y & -0.00086331 & -0.00007474 & 0.00000234 \\
\hline Sec. Sph & 0.84480124 & 0.87833575 & 0.77203126 \\
\hline $\begin{array}{c}\text { Overall } \\
\text { RMS }\end{array}$ & $5.37 \mu \mathrm{m}$ & $5.79 \mu \mathrm{m}$ & $4.93 \mu \mathrm{m}$ \\
\hline
\end{tabular}




\section{RESULTS}

\subsection{Results of Loadcases to Find the Optimum SMP}

\section{Approach}

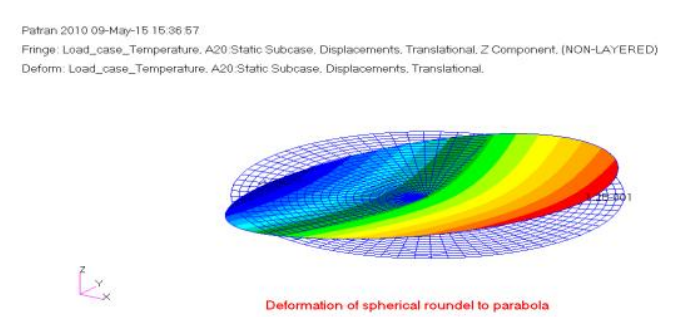

Fig-12: LC: $1-(7.711 \mu \mathrm{m})$

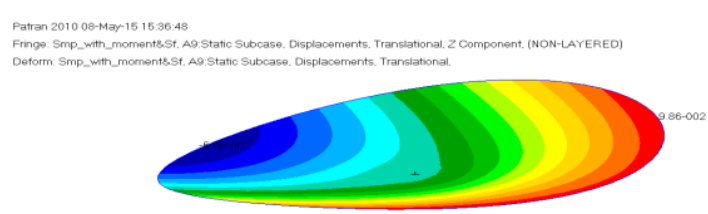

Fig-13: LC: $2-(11.866 \mu \mathrm{m})$
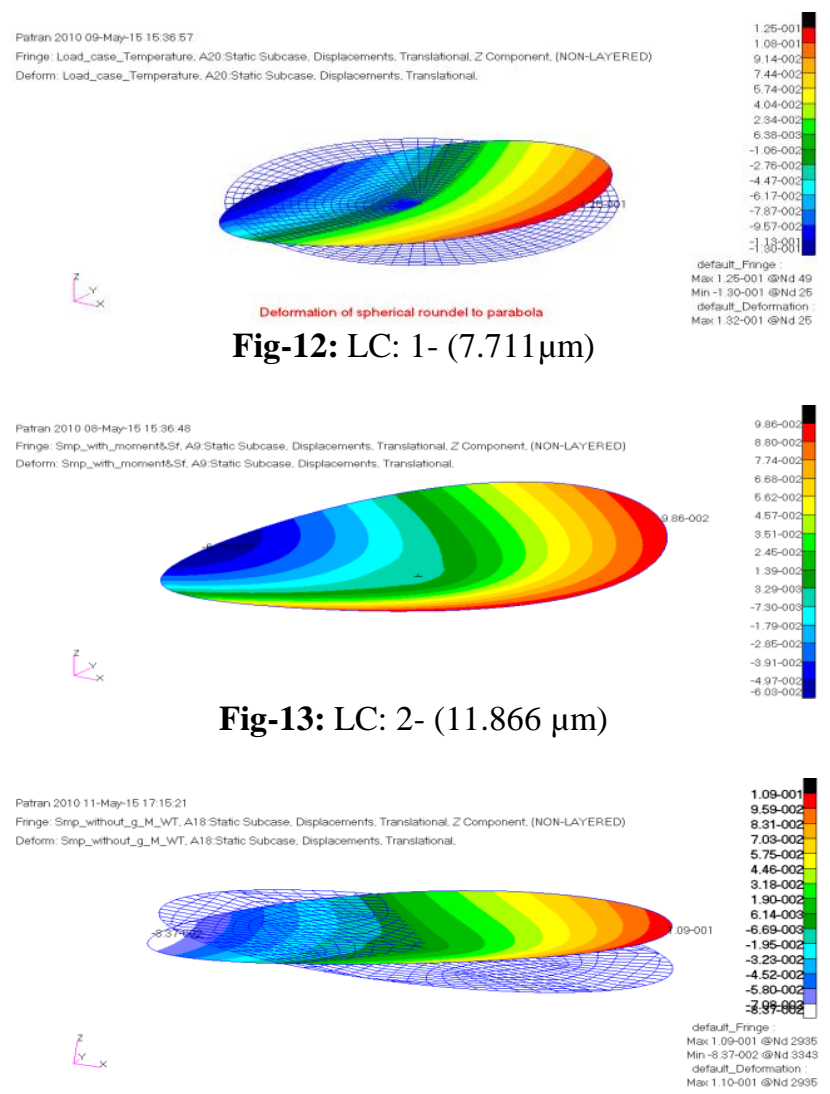

Fig-14: LC: $3 . \mathrm{a}-(5.6824 \mu \mathrm{m})$

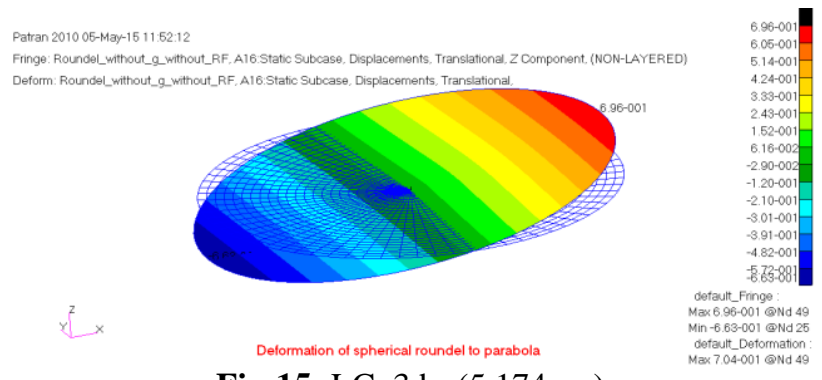

Fig-15: LC: 3.b- $(5.174 \mu \mathrm{m})$

Front Surface Deformation on the Spherical Mirror due to different load cases given in microns.

\subsection{Results of Exercises To Prove Reversibility of} SMP

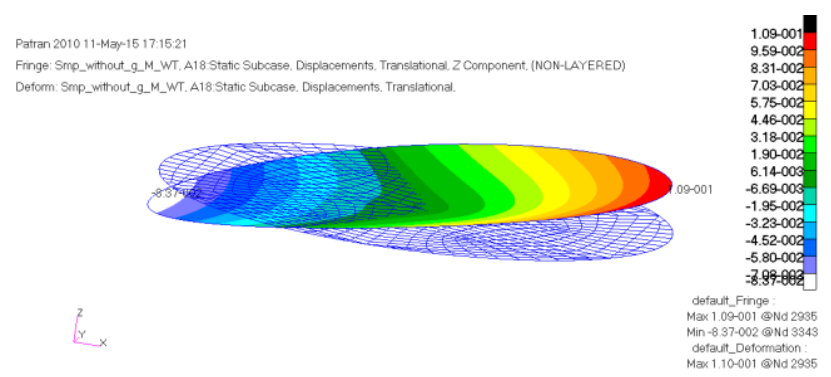

Fig-16: Spherical to Parabola $(5.6824 \mu \mathrm{m})$

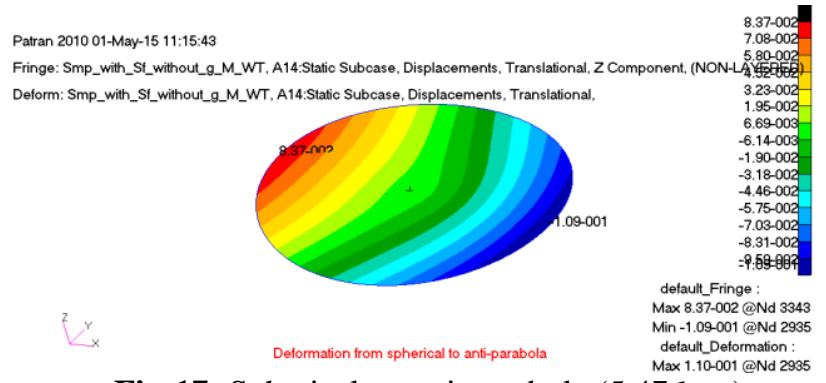

Fig-17: Spherical to anti-parabola $(5.476 \mu \mathrm{m})$

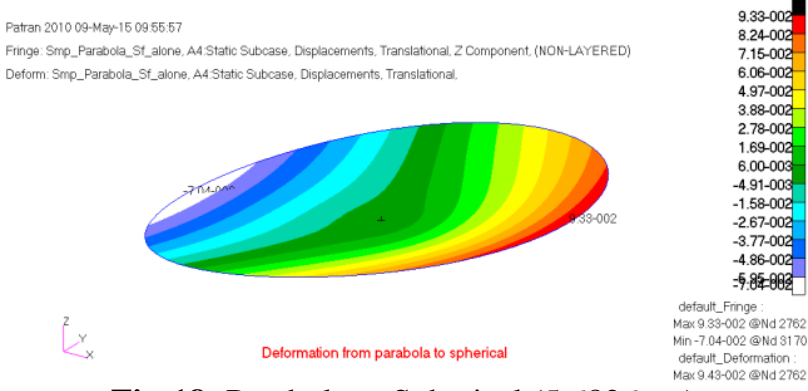

Fig-18: Parabola to Spherical $(5.6826 \mu \mathrm{m})$

\section{CONCLUSION}

This paper describes the study on Opto-mechanical design and FE analysis for force calculation. The purpose of the work is to simulate the deformed surface of parabola after forcing it and comparing with an ideal best fit spherical using different approach and to find out an optimum one.

$>$ Among the all loading conditions, the Load case with shear force and central support is found to be the optimum approach.

$>$ The surface figure of 5.18 microns is achieved through the shear force and central support alone.

> With same loads and boundary conditions, the RMS surface figure after the deformation of spherical to parabola and parabola to spherical is found to be $5.6824 \mu \mathrm{m}$ and $5.6826 \mu \mathrm{m}$ respectively $(0.2 \mathrm{~nm}$ deviation), and spherical to anti-parabola is $5.476 \mu \mathrm{m}$, all of these prove the reversibility of the SMP.

$>$ The same model for various thicknesses is re-modeled and analyzed by calculating the forces, to validate the calculations.

$>$ By proper modification of the applied shear force, the surface figure is improved from 5.37 microns to 4.9 microns. So further modification is also possible by the correction of selected aberrations.

\section{ACKNOWLEDGEMENTS}

The authors gratefully acknowledge the support of Indian Institute of Space Science and Technology (IIST), Thiruvananthapuram and Laboratory for Electro-Optics Systems (LEOS-ISRO), Bangalore.

\section{REFERENCES}

[1] Lubliner and Nelson, "Stressed Mirror Polishing. 1: A technique for producing non-axisymmetric mirrors", TMT Report No. 61. [Applied Optics, Vol. 19, No. 14, 15 July 1980]. (Keck Observatory Report No. 21) 
[2] Nelson, Gabor, Hunt, Lubliner, Mast, "Stressed Mirror Polishing. 2: Fabrication of an off-axis section of a paraboloid", TMT Report No. 62. [Applied Optics, Vol. 19, No. 14, 15 July 1980]. (Keck Observatory Report No. 22)

[3] Nelson and Temple-Raston, "The Off-Axis Expansion of Conic Surfaces", UC TMT (Keck) Report No. 91, November 1982

[4] Terry S. Mast and Jerry E. Nelson [1985]. Fabrication of Keck off-axis mirror segments. SPIE Vol. 542.

[5] Stephen F. Sporer, "TMT - Stressed Mirror Polishing Fixture Study", Tinsley Laboratories, Division of SSG Precision Optronics,4040 Lakeside Drive, Richmond, CA 94806, USA

[6] Paul. R. Yoder, Jr. "Opto-Mechanical Systems Design" Third Edition, 2006, SPIE Press.

[7] Anees Ahmed, "Handbook of Opto-mechanical Engineering" 1997 CRC Press.

\section{BIOGRAPHIES}

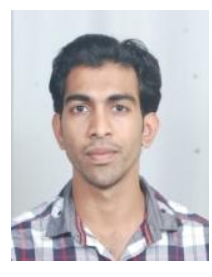

Alikhan Basheer received the B-Tech degree in Electronics and Communication Engineering from the Mahatma Gandhi University, Kottayam, India, in 2012 and the M-Tech degree in Optical Engineering from the Indian Institute of Space Science and Technology (IIST), Thiruvananthapuram, India, in 2015.His research is focused on Opto-mechanical design and analysis of mechanical fixtures for TMT primary mirror segments. His research also includes the metrology study for the TMT Primary mirrors.

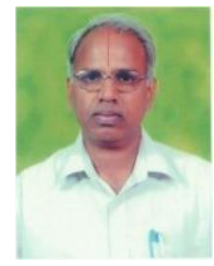

KrishnaMurthy T received B.Sc. degree in physics from Osmania Univeristy in 1974 and the M.Sc (Tech) Engineering Physics degree (with specialization in Modern and Applied Optics) from NIT Warangal (Kakatiya University), in 1977. With an experience of 30 years in Indian Space Research Organization (ISRO), he is currently teaching Opto Mechanical Engineering \& Adaptive Optics to Masters Degree students of Indian Institute of Space Science and Technology (IIST), Thiruvananthapuram since 2012. 